\title{
Purely electromagnetic spacetimes
}

\author{
B.V.Ivanov* \\ Institute for Nuclear Research and Nuclear Energy, \\ Bulgarian Academy of Sciences \\ Tzarigradsko Shausse 72, Sofia 1784, Bulgaria
}

\begin{abstract}
Electrovacuum solutions devoid of usual mass sources are classified in the case of one, two and three commuting Killing vectors. Three branches of solutions exist. Electromagnetically induced mass terms appear in some of them.

PACS numbers: 04.40.Nr
\end{abstract}

\section{INTRODUCTION}

In general relativity electromagnetic fields alter the metric of spacetime through their energy-momentum tensor

$$
T_{\nu}^{\mu}=-\frac{1}{4 \pi}\left(F^{\mu \alpha} F_{\nu \alpha}-\frac{1}{4} \delta_{\nu}^{\mu} F^{\alpha \beta} F_{\alpha \beta}\right)
$$

where

$$
F_{\mu \nu}=\partial_{\mu} A_{\nu}-\partial_{\nu} A_{\mu}
$$

is the electromagnetic tensor and $A_{\mu}$ is the four-potential. $T_{\nu}^{\mu}$ enters the r.h.s. of the Einstein equations

$$
R_{\nu}^{\mu}=-8 \pi T_{\nu}^{\mu}
$$

We use relativistic units $G=c=1$ and take into account that $T_{\mu \nu}$ is traceless. In addition, the Maxwell equations are coupled to gravity through the covariant derivatives of $F_{\mu \nu}$

$$
F_{; \nu}^{\mu \nu}=\frac{1}{\sqrt{-g}}\left(\sqrt{-g} F^{\mu \nu}\right)_{\nu}=0 .
$$

Here $g$ denotes the metric's determinant and we discuss electrovacuum solutions. The Einstein-Maxwell (EM) equations $(3,4)$ show how the electromagnetic field leaves its imprint on the metric.

Already in 1925 Rainich gave the necessary and sufficient conditions for a gravitational field to originate from a nonnull electromagnetic field [1], 2], [3]. They split into an algebraic and an analytic part, which involve the Ricci tensor and determine the associated electromagnetic field up to a constant duality rotation. This method is cumbersome for finding exact solutions, but is useful to check those already found [3]. In fact, it is very hard to find any exact solution for a completely general metric. One has to introduce some symmetry, study algebraically special fields or fields containing special vectors and tensors. This seems to simplify the Rainich conditions too. Thus, in Ref. [4] it is shown that type D aligned EM solutions are characterized just by algebraic restrictions on $R_{\mu \nu}$.

In this paper we study the effect on spacetime of electromagnetic fields possessing some symmetry. The metric inherits this symmetry and has Killing vectors which commute and increase from one to three. Hence, it depends on three, two and one coordinates.

When the electromagnetic field is turned off Eq (4) becomes trivial, while Eq (3) transforms into the vacuum Einstein equations. They have a number of non-trivial solutions besides flat Minkowski spacetime. Usually singularities are present where hidden mass sources lie. Thus the Schwarzschild solution, although being a vacuum one, represents the field of a point mass, sitting at the origin. We want to study the pure electromagnetic effect on the metric, therefore we demand that no traditional masses are present and when the electromagnetic field is turned off, flat spacetime results. We call such spacetimes purely electromagnetic spacetimes (PES).

In Section II stationary solutions are discussed by introducing the Ernst potential, the EM equations based on it and their group of symmetry transformations. It is shown that PES are characterized by a real constant taking three

*Electronic address: boyko@inrne.bas.bg 
values. The metric components are expressed through the electromagnetic potential, which satisfies one fundamental equation with three branches of solutions. Sometimes linear terms in the main metric function appear, leading to electromagnetically induced mass terms.

In Section III static PES with one Killing vector are further elaborated. There is a large class of solutions based on a harmonic function.

In Section IV stationary axisymmetric PES are discussed with emphasis on their multipole structure.

In Section $\mathrm{V}$ the static subcase is studied and the emergence of Weyl solutions is clarified.

Section VI is dedicated to PES with plane and cylindrical symmetry. All such spacetimes are found explicitly, based on a simple harmonic function.

Section VII contains conclusions and discussion.

\section{STATIONARY PES}

Let the metric possess one Killing vector, taken time-like for convenience. Such gravitational fields are called stationary [3], [5] and the interval reads

$$
d s^{2}=f\left(d x^{0}+\omega_{m} d x^{m}\right)^{2}-f^{-1} \gamma_{m n} d x^{m} d x^{n} .
$$

The metric components are independent of time. There exists an Ernst potential $E$ [6]

$$
E=f-\Psi \Psi^{*}+i \psi, \quad \Psi=\phi+i \lambda,
$$

where the scalar electric and magnetic potentials are defined as

$$
F_{0 n}=\phi_{, n}, \quad F^{m n}=f \gamma^{-1 / 2} \varepsilon^{m n p} \lambda_{, p}
$$

The three-dimensional metric $\gamma_{m n}$ determines a corresponding vector calculus and $\gamma$ is its determinant, while the comma denotes a derivative. The imaginary part of $E$ follows from $\omega_{m}, \Psi$ and $f$

$$
\begin{gathered}
\nabla \psi=\vec{\tau}+i\left(\Psi^{*} \nabla \Psi-\Psi \nabla \Psi^{*}\right), \\
f^{-2} \tau^{m}=-\gamma^{-1 / 2} \varepsilon^{m p q} \partial_{p} \omega_{q} .
\end{gathered}
$$

The existence of the Ernst potential leads to considerable simplification of the EM equations for $E$, $\Psi$ and $\gamma_{m n}$

$$
\begin{gathered}
f \nabla^{2} E=\nabla E\left(\nabla E+2 \Psi^{*} \nabla \Psi\right), \\
f \nabla^{2} \Psi=\nabla \Psi\left(\nabla E+2 \Psi^{*} \nabla \Psi\right), \\
-f^{2} R_{m n}(\gamma)=\frac{1}{2} E_{,(m} E_{, n)}^{*}+\Psi E_{,(m} \Psi_{, n)}^{*}+\Psi^{*} E_{,(m}^{*} \Psi_{, n)}-\left(E+E^{*}\right) \Psi_{,(m} \Psi_{, n)}^{*} .
\end{gathered}
$$

Symmetrization is meant on the r.h.s. of the last equation, while $f$ is given by $\operatorname{Eq}(6)$

$$
f=\frac{1}{2}\left(E+E^{*}\right)+\Psi \Psi^{*}
$$

It is well-known that the group of symmetry transformations of these equations is $S U(2,1)$ [7]. A non-linear representation of it consists of 5 transformations containing 3 complex and 2 real parameters [3], p 520. We shall use two of them that do not change the solution in a non-trivial manner, namely

$$
\begin{aligned}
& E^{\prime}=E+i b, \quad \Psi^{\prime}=\Psi, \\
& E^{\prime}=\alpha \alpha^{*} E, \quad \Psi^{\prime}=\alpha \Psi,
\end{aligned}
$$

while $\gamma_{m n}$ is not transformed. Here $\alpha$ is a complex parameter, while $b$ is real. The first transformation is a gauge one. The second is a duality rotation when $|\alpha|=1$ and a rescaling of $d s$ otherwise. 
A linear representation of the group of motions is given as follows [7]. The Ernst and the electromagnetic potentials are parameterized by 3 complex scalar fields $u, q, w$

$$
E=\frac{u-w}{u+w}, \quad \Psi=\frac{q}{u+w} .
$$

One of them $(w)$ is redundant and is chosen so that Eqs $(10,11)$ become

$$
\left(u u^{*}+q q^{*}-w w^{*}\right) \nabla^{2} Z=2\left(u^{*} \nabla u+q^{*} \nabla q-w^{*} \nabla w\right) \nabla Z,
$$

where $Z=u, q$ or $w$. One can further set $u=1, w=\xi$ or $u=\xi, w=1$ obtaining

$$
E=\frac{1-\xi}{1+\xi} \quad \text { or } \quad E=\frac{\xi-1}{\xi+1}, \quad \Psi=\frac{q}{1+\xi} .
$$

The potential $\Psi$ is defined up to a constant $c$. We demand that when $\Psi \rightarrow c$ Minkowski spacetime should result. This means that $\omega_{m}=0$ and Eqs $(8,9)$ yield $\psi=0$. Obviously $f$ should become equal to unity. Then Eq $(6)$ gives $E=1-c c^{*}$, which is a real constant, containing no mass parameters. When $\Psi$ is turned on, $E$ remains constant for PES. This is because the real and imaginary part of $\xi$ give the mass and rotation potentials in the multipole structure of the solution [8], hence, for PES $\xi$ and correspondingly $E$ should be trivial constants. We can make them real by transformation (14). Using $\mathrm{Eq}(15)$ the real constant $E_{0}$ can be set to one of the 3 distinct values $1,0,-1$. Hence, there are three branches of PES. In a similar way, the inequivalent classes of solutions under the action of the complete $S U(2,1)$ group are given by $E=1,0,-1$ and $E=E^{*}[3]$, Fig. 34.1.

The setting of $E=E_{0}, \psi=0$ for PES causes drastic simplification in the EM equations. Eq (10) becomes trivial, $\mathrm{Eq}$ (13) gives

$$
f=E_{0}+\Psi \Psi^{*}
$$

The quadratic dependence of $f$ on $\Psi$ can be traced to the quadratic dependence of the energy-momentum tensor on $F_{\mu \nu}$. Inserting Eq (19) into Eq (11) one gets an equation for $\Psi$

$$
\left(\Psi \Psi^{*}+E_{0}\right) \nabla^{2} \Psi=2 \Psi^{*} \nabla \Psi \nabla \Psi .
$$

Next, $\omega_{q}$ is determined from Eqs $(8,9)$

$$
f^{2} \gamma^{-1 / 2} \varepsilon^{m p q} \partial_{p} \omega_{q}=i\left(\Psi^{*} \nabla \Psi-\Psi \nabla \Psi^{*}\right)=i \Psi \Psi^{*} \nabla \ln \frac{\Psi}{\Psi^{*}} .
$$

Eq (12), which determines the three-metric $\gamma_{m n}$ simplifies considerably

$$
f^{2} R_{m n}(\gamma)=2 E_{0}\left(\Psi_{, m} \Psi_{, n}^{*}+\Psi_{, n} \Psi_{, m}^{*}\right)
$$

The 3 branches of the solution are marked by different values of $c=c_{1}+i c_{2}$

$$
c_{1}^{2}+c_{2}^{2}=1-E_{0} .
$$

Let us define the potential $\Psi_{1}=\phi_{1}+i \lambda_{1}=\Psi-c$ so that $\Psi_{1} \rightarrow 0$ always when the electromagnetic field is turned off. Eq (22) remains the same but with $\Psi$ replaced by $\Psi_{1}$. Eq (19) becomes

$$
f=1+2 c_{1} \phi_{1}+2 c_{2} \lambda_{1}+\phi_{1}^{2}+\lambda_{1}^{2}
$$

There are obviously linear terms in $f$ whenever $c \neq 0$, i.e. $E_{0} \neq 1$.

Neither of the parameterizations (18) can encompass all three branches of the solution, but this is possible in the parameterization (16)

$$
\begin{aligned}
& E_{0}=-1 ; \quad u=0, w=1, \Psi=q, \\
& E_{0}=0 ; \quad u=w=1, \Psi=q / 2, \\
& E_{0}=1 ; \quad u=1, w=0, \Psi=q .
\end{aligned}
$$


In all three cases $\mathrm{Eq}(17)$ becomes identical to $\mathrm{Eq}(20)$. When $E_{0}=-1 \mathrm{Eq}(20)$ coincides in form with the vacuum Ernst equation for $\xi$ replaced by $q$. When $E_{0}=1$ this equation has been derived also by Tanabe [9] from the condition for a linear relation between $u, q, w$. When $E_{0}=0 \mathrm{Eq}(20)$ is equivalent to

$$
\Psi=H^{-1}, \quad \nabla^{2} H=0 .
$$

We also get $R_{m n}(\gamma)=0$, so that space turns flat, $H$ decouples from $\gamma_{m n}$ and becomes an arbitrary complex harmonic function. Furthermore

$$
f=\frac{1}{H H^{*}}, \quad \varepsilon^{m p q} \partial_{p} \omega_{q}=i H H^{*} \nabla \ln \frac{H^{*}}{H} .
$$

Let $H=L+i M$, where $L$ and $M$ are real harmonic functions. Then

$$
\begin{gathered}
f=\frac{1}{L^{2}+M^{2}}, \quad \Psi=\frac{1}{L+i M}, \\
\phi=\frac{L}{L^{2}+M^{2}}=f L, \quad \lambda=-\frac{M}{L^{2}+M^{2}}=-f M .
\end{gathered}
$$

Electromagnetic fields alone are unable to induce strong gravitational fields like those around black holes, therefore $f \approx 1$ and $\phi, \lambda$ are almost harmonic, as follows from Eq (31). At infinity a monopole term in $\phi, \phi \sim e / R$ where $e$ is the charge and $R^{2}=r^{2}+z^{2}$, will induce a long-range mass-type term in $f$ according to Eq (24)

$$
f \sim 1+2 c_{1} \frac{e}{R}
$$

as long as $c_{1} \neq 0\left(E_{0} \neq 1\right)$. It presents an electromagnetically induced mass. Otherwise the gravitational field of PES remains short-ranged.

Eq (23) does not determine the signs of $c_{1}$ and $c_{2}$. One can choose them in such a way that the induced mass is always positive, no matter what the sign of the charge $e$ is.

The formulas for the $E_{0}=0$ branch resemble those of Perjés-Israel-Wilson (PIW) fields [5], [10], 11] but their physical interpretation is completely different. PIW solutions are derived upon the condition of flat space metric, which leads to a linear relation between $E$ and $\Psi[5]$

$$
\Psi=\frac{1}{2}(1-E) .
$$

A comparison with $\mathrm{Eq}(18)$ gives $\xi=q$. Thus $\xi$ is not trivial and there are rotating masses in the system, besides the electromagnetic field. In fact, the masses of the sources are equal to the charges $m_{i}=e_{i}$. Obviously such solutions are not PES. Curiously, PIW metrics also become flat when $\Psi$ is turned off, but this is ensured by taking away some mass in order to keep the relations $m_{i}=e_{i}$ intact, even when $e_{i} \rightarrow 0$.

\section{STATIC PES WITH ONE KILLING VECTOR}

Stationary gravitational fields are static when the Killing vector is hyper-surface orthogonal, $\omega_{m}=0$. Let us write

$$
\Psi=\phi+i \lambda=\chi \cos \delta+i \sin \delta
$$

Then due to Eq $(21) \delta$ is constant and $\phi \sim \lambda$. The effects of electric and magnetic fields on gravity are identical and we put for simplicity $\lambda=0$. The same conclusion holds for general static fields [12], [13], [14]. This choice makes $\Psi$ real and equal to $\phi$. One can always maintain that it depends on the polar coordinates $r, z, \varphi$ through some other function $h$. Eq (20) becomes

$$
\left[\left(\phi^{2}+E_{0}\right) \phi_{h h}-2 \phi \phi_{h}^{2}\right] \nabla h \nabla h=-\left(\phi^{2}+E_{0}\right) \phi_{h} \nabla^{2} h .
$$

A large class of solutions may be found when $h$ is harmonic. Then Eq (35) can be integrated

$$
h=\int \frac{d \phi}{\phi^{2}+E_{0}} .
$$


The integral has 3 analytic expressions according to the value of $E_{0}$ and in all of them the functional dependence $h(\phi)$ can be inverted

$$
\begin{array}{cc}
E_{0}=0, & \phi=-1 / h, \\
E_{0}=1, \quad \phi=\tan h, \\
E_{0}=-1, \quad \phi=\frac{1+e^{2 h}}{1-e^{2 h}} .
\end{array}
$$

In the last case we took into account that $c=\sqrt{2}>1$. The denominator in Eq (36) is in fact $f$, which leads to the relation $\phi_{, i}=f h_{, i}$ similar to Eq (31). Thus $\phi$ is almost harmonic. Eq (22) becomes

$$
R_{m n}(\gamma)=\frac{4 E_{0} \phi_{m} \phi_{n}}{\left(\phi^{2}+E_{0}\right)^{2}}
$$

In the branch $E_{0}=0$ space is flat, $h$ decouples from $\gamma_{m n}$ and the solution resembles the Majumdar-Papapetrou (MP) solutions [15], [16], but still the physical interpretation is different. The latter solutions are a static subcase of the PIW solutions and their sources have masses equal to the charges.

\section{STATIONARY AXISYMMETRIC PES}

Such metrics possess two commuting Killing vectors and do not depend on $t$ and $\varphi$. The interval is

$$
d s^{2}=f(d t-\omega d \varphi)^{2}-f^{-1}\left[e^{2 k}\left(d r^{2}+d z^{2}\right)+r^{2} d \varphi^{2}\right]
$$

The solutions with $E_{0}= \pm 1$ in the previous sections are inexplicit because $\nabla$ and $\nabla^{2}$ depend on $\gamma_{m n}$, which depends in turn on $\Psi$ through Eqs $(12,22,40)$. This vicious circle breaks for all axisymmetric solutions; the gradient and the Laplacian are the flat 3-dimensional ones. Eq (20) decouples from $k$. The same is true for Eq (21)

$$
\frac{f^{2}}{r} \omega_{r}=2\left(\phi \lambda_{z}-\lambda \phi_{z}\right), \quad \frac{f^{2}}{r} \omega_{z}=2\left(\lambda \phi_{r}-\phi \lambda_{r}\right) .
$$

Eq (19) is unchanged, while Eq (22) simplifies to

$$
\begin{gathered}
k_{r}=\frac{1}{2} r\left(R_{r r}-R_{z z}\right)=\frac{2 E_{0} r}{f^{2}}\left(\Psi_{r} \Psi_{r}^{*}-\Psi_{z} \Psi_{z}^{*}\right) \\
k_{z}=r R_{r z}=\frac{2 E_{0} r}{f^{2}}\left(\Psi_{r} \Psi_{z}^{*}+\Psi_{z} \Psi_{r}^{*}\right)
\end{gathered}
$$

The equations for $\omega$ and $k$ are linear and can be easily solved after $\Psi$ is found from Eq (20).

When $E_{0}=0$ Eqs (28-31) still hold and together with Eq (42) give the PES solution. As an example, let us choose $L$ and $M$ as

$$
\begin{gathered}
L=1+\frac{e}{R}, \quad M=\frac{\mu z}{R^{3}}, \\
\Psi=\frac{R^{3}}{R^{3}+e R^{2}+i \mu z} .
\end{gathered}
$$

Then near infinity we have

$$
\phi \sim 1-\frac{e}{R}, \quad \lambda \sim-\frac{\mu z}{R^{3}}, \quad f \sim 1-\frac{2|e|}{R},
$$

so that the solution has charge $-e$, magnetic moment $-\mu$ and a monopole term of purely electromagnetic origin in $f$, corresponding to a mass equal to $|e|$. The angular momentum is $-\mu$ and is of magnetic origin. This solution represents a massive charged magnetic dipole, whose mass and angular momentum are induced electromagnetically. 
There are different techniques for solving the EM equations in the general axisymmetric case. One of them is the Sibgatullin-Manko method, based on a prescribed behavior of the Ernst potential on the symmetry axis [17], 18]. One starts with

$$
E(r=0, z)=1+\sum_{l=1}^{N} \frac{\alpha_{l}}{z-\beta_{l}}
$$

and a similar expression for $\Psi$, where $\alpha_{l}, \beta_{l}$ are given constant parameters related to the mass, angular momentum and higher multipole moments. The solution is obtained by a sophisticated integration procedure. It is characterized by the gravitational and electromagnetic moments $P_{i}$ and $Q_{i}[8],[19]$. They are determined from the coefficients $\xi_{i}, q_{i}$ of the series expansion of $\xi$ and $q$ near infinity. The infinite point is brought to the origin of the coordinates by a conformal transformation $(r, z) \rightarrow(\bar{r}, \bar{z})$. One can write

$$
\xi(\bar{r}=0, \bar{z})=\sum_{i=0}^{\infty} \xi_{i} \bar{z}^{i+1}, \quad q(\bar{r}=0, \bar{z})=\sum_{i=0}^{\infty} q_{i} \bar{z}^{i+1},
$$

where $\bar{z}=1 / z$. Eq (48) shows that PES is obtained as a special massless case when $\alpha_{l}=\beta_{l}=0$ and this gives always a member of the $E_{0}=1$ branch. The same conclusion follows from Eq (49); $\xi$ becomes trivial only when all $\xi_{i}=0$. Then $\xi=0$ and $E=1$. The massless case has been discussed in a number of papers [20], 21], [22], 223]. In the last reference the explicit solution for a massless magnetic dipole is presented. One can check that in all these examples $E=1$ and therefore $c=0$. There are no linear terms in $f$ and no electromagnetically induced mass.

It must be pointed out that the expansion in Eq (49) cannot include $\xi=1$ and consequently the $E_{0}=0$ branch of PES. This happens because there is no constant term in it. On the other side PIW solutions have $\xi_{i}=q_{i}$ and do fit into Eq (49). In addition, they are massive, not massless solutions.

The branch $E_{0}=-1$ has $\Psi=q$ and $\mathrm{Eq}$ (20) is equivalent to the vacuum Ernst equation for $q$ instead of $\xi$. Its solutions can be found by the Sibgatullin-Manko method and many other methods [3]. The metric components $f, \omega$ and $k$ are given by Eqs $(19,42-44)$.

\section{STATIC AXISYMMETRIC PES}

Based on the previous sections one can describe these spacetimes as follows. The function $\omega$ vanishes and $\phi \sim \lambda$. We put $\lambda=0$ for simplicity. Eq (20) becomes

$$
\left(\phi^{2}+E_{0}\right) \nabla^{2} \phi=2 \phi \nabla \phi \nabla \phi
$$

where the differential operators are flat and 3-dimensional. Next

$$
f=1+2 c_{1} \phi_{1}+\phi_{1}^{2}=E_{0}+\phi^{2}
$$

where $E_{0}=1-c_{1}^{2}, \phi=\phi_{1}+c_{1}$ and $\phi_{1} \rightarrow 0$ when the electric field is turned off and also at infinity. Finally

$$
k_{r}=\frac{2 E_{0} r}{f^{2}}\left(\phi_{r}^{2}-\phi_{z}^{2}\right), \quad k_{z}=\frac{4 E_{0} r}{f^{2}} \phi_{r} \phi_{z} .
$$

The class of solutions given by Eq (36) is still valid, $h$ being an arbitrary real harmonic function. It was discovered by H.Weyl in 1917 [24] and includes the three branches (37-39). The branch $E_{0}=0$ is exhausted by Weyl solutions and is conformastatic, but for $E_{0}= \pm 1$ there can be solutions of Eq (50) not based on a harmonic function. Similarly to the stationary axisymmetric PES with $E_{0}=1$, static axisymmetric PES with this property are among the massless cases of static electrovacuum Sibgatullin-Manko solutions [25]. There are of course electrovacuum solutions containing usual mass sources, but the quadratic relation in Eq (51) breaks for them in principle.

Weyl solutions are usually derived by demanding functional dependence $f\left(\phi_{1}\right)$ which leads to $f_{\phi_{1} \phi_{1}}=2$ and leaves the coefficient $c_{1}$ in Eq (51) undetermined. We see that it descends from the Ernst potential $E_{0}$ of the solution and is fixed to one of three distinct values $0,1, \sqrt{2}$.

\section{PES WITH 3 COMMUTING KILLING VECTORS}

Such metrics depend on just one coordinate; $z$ (plane symmetry) or $r$ (cylindrical symmetry). More generally, they can depend on some function of $r, z$ and spherical symmetry is also included, but we shall not discuss this possibility 
in the present paper. Eq (42) tells us that when $\Psi=\Psi(z)$ it induces $\omega(r)$ and vice versa. The symmetry of the electromagnetic field is inherited only when $\omega=0$ and the solution is static. There are stationary cylindrical solutions like the one given by Eq (22.17) from Ref [3] but it doesn't transfer into Minkowski spacetime when the electric field is turned off and is not a PES. Now $\phi$ is a function of either $z$ or $r$ and consequently is a function of either $h=1-Q z$ or $h=1-Q \ln r$ which are harmonic. Thus Eq (20) transforms into Eq (36) and its 3 branches comprise all solutions. Eq (52) becomes

$$
k_{r}=2 E_{0} r\left(h_{r}^{2}-h_{z}^{2}\right), \quad k_{z}=4 E_{0} r h_{r} h_{z}
$$

Let us discuss first the plane-symmetric case. We have $k_{z}=0$ and

$$
k=-E_{0} Q^{2} r^{2}
$$

The plane symmetry of the electric field is not inherited by the metric unless the branch $E_{0}=0$ is chosen. Then $k=0$ and

$$
\phi=\frac{1}{1-Q z}, \quad f=(1-Q z)^{-2} .
$$

This is written in the form of Eq (51) like

$$
f=1+2 \phi_{1}+\phi_{1}^{2}, \quad \phi_{1}=\phi-1,
$$

so that when $Q=0$ we get $\phi_{1}=0$ and $f=1$. There is clearly a linear term in $f$.

The electrified plane-symmetric solution has been studied by many authors, working in different coordinate systems. It appeared for the first time in a paper by Kar [26]. The case with $k=0$ was singled out by McVittie [27].

In the cylindrical case one should replace $z$ by $\ln r$ in Eq (55). The function $k=k(r)$ again, but now all three branches preserve cylindrical symmetry. The one with $E_{0}=0$ is the metric given by Eq (22.16) from [3] after some corrections are made. It was found by Bonnor [28] and rederived by Raychaudhuri [29] who used the Rainich formalism.

\section{CONCLUSIONS AND DISCUSSION}

We have shown in this paper that the Rainich program of describing electromagnetically induced metrics can be implemented in a simpler way and in more detail when the symmetry of the system is gradually increased. PES require that the Ernst potential becomes a constant with 3 possible values. This invokes a quadratic dependence of the main metric function $f$ on the electromagnetic potential $\Psi$ and its complex conjugate. The two basic field equations $(10,11)$ reduce to a single one $\mathrm{Eq}(20)$ for $\Psi$. It possesses 3 branches of solutions - one harmonic, one of Ernst type and one of quasi-Ernst type. In some cases $f$ has linear in $\Psi_{1}$ terms, which give rise to electromagnetically induced mass terms. Solutions in harmonic functions form a large class when one or two Killing vectors are present and become exhaustive when the metric depends on a single coordinate. We have also shown that the 3-branched Weyl solution is a kind of PES and has analogs in axisymmetric and stationary fields.

There are many EM solutions in the literature and it seems strange that PES were not studied systematically in the past. The reason probably is that vacuum solutions are studied first as being simpler. Then they are electrified and magnetised. In this way traditional mass sources co-exist with the electromagnetic field and the resulting gravitation is a mixture due to the both types of sources. In other areas of general relativity the corresponding problem has already been investigated.

In the case of charged perfect fluids there is a bunch of spherically symmetric models where mass arises in a purely electromagnetic way [30], [31]. Some of them were proposed as classical models of the electron.

The study of the gravitation of beams of incoherent light, whose energy-momentum tensor is of pure radiation type, began already in 1931 in the linear approximation [32]. Later Bonnor found exact solutions belonging to the class of pp-waves [33]. Even the gravitational field of two identical colliding beams of light was found recently [34]. Although these fields are rather weak, such studies have important conceptual motive to unravel the non-linear structure of general relativity.

The sources of PES are point mixtures of electric and magnetic multipoles without any mass multipoles. There should also be regular PES arising from rotating charged and magnetised surfaces. In this respect they are completely 
different from PIW and MP solutions whose sources are massive and represent extreme black holes or shells of charged dust balanced by masses equal to the charges [35], [36], [37].

[1] G.Y.Rainich, Trans. Amer. Math. Soc. 27, 106 (1925)

[2] C.W.Misner and J.A.Wheeler, Ann. Phys. 2, 525 (1957)

[3] H.Stephani, D.Kramer, M.Maccallum, C.Hoenselaers and E.Herlt, Exact solutions to Einstein's field equations, Second edition (Cambridge University Press, Cambridge, 2003)

[4] J.Y.Ferrando and J.A.Sáez, preprint, arXiv: gr-qc/0703049 (2007)

[5] W.Israel and G.A.Wilson, J. Math. Phys. 13, 865 (1972)

[6] F.J.Ernst, Phys. Rev. 168, 1415 (1968)

[7] W.Kinnersley, J. Math. Phys. 14, 651 (1973)

[8] C.Hoenselaers and Z.Perjés, Class. Quantum Grav. 7, 1819 (1990)

[9] Y.Tanabe, Prog. Theor. Phys. 57, 840 (1977)

[10] Z.Perjés, Phys. Rev. Lett. 27, 1668 (1971)

[11] W.B.Bonnor and J.P.Ward, Commun. Math. Phys. 28, 323 (1972)

[12] Z.Perjés, Nuovo Cim. B 55, 600 (1968)

[13] A.Das, J. Math. Phys. 20, 740 (1979)

[14] B.V.Ivanov, Class. Quantum Grav. 13, 303 (1996)

[15] S.D.Majumdar, Phys. Rev. 72, 390 (1947)

[16] A.Papapetrou, Proc. R. Irish Acad. A 51, 191 (1947)

[17] V.S.Manko and N.R.Sibgatullin, Class. Quantum Grav. 10, 1383 (1993)

[18] E.Ruiz, V.S.Manko and J.Martin, Phys. Rev. D 51, 4192 (1995)

[19] T.P.Sotiriou and T.A.Apostolatos, Class. Quantum Grav. 21, 5727 (2004)

[20] V.S.Manko and N.R.Sibgatullin, Phys. Lett. A 168, 343 (1992)

[21] V.S.Manko and N.R.Sibgatullin, Class. Quantum Grav. 9, L87 (1992)

[22] V.S.Manko and N.R.Sibgatullin, J. Math. Phys. 34, 170 (1993)

[23] V.S.Manko et al, Phys. Rev. D 49, 5144 (1994)

[24] H.Weyl, Ann. Phys.(Germany) 54, 117 (1917)

[25] N.Breton, V.S.Manko and J.A.Sánchez, Class. Quantum Grav. 15, 3071 (1998)

[26] S.G.Kar, Physik. Zeitschr. 27, 208 (1926)

[27] G.C.McVittie, Proc. R. Soc. A 124, 366 (1929)

[28] W.B.Bonnor, Proc. Phys. Soc. A 66, 145 (1953)

[29] A.K.Raychaudhuri, Ann. Phys. 11, 501 (1960)

[30] R.N.Tiwari, J.R.Rao and R.R.Kanakamedala, Phys. Rev. D 30, 489 (1984)

[31] B.V.Ivanov, Phys. Rev. D 65, 104001 (2002)

[32] R.C.Tolman, P.Ehrenfest and B.Podolsky, Phys. Rev. 37, 602 (1931)

[33] W.B.Bonnor, Commun. Math. Phys. 13, 163 (1969)

[34] B.V.Ivanov, Class. Quantum Grav. 20, 397 (2003)

[35] M.Gürses, Phys. Rev. D 58, 044001 (1998)

[36] V.Varela, Gen. Relat. Grav. 35, 1815 (2003)

[37] M.Gürses and B.Himmetoglu, Phys. Rev. D 72, 024032 (2005) 